\title{
Metodología basada en el enfoque de redes y uso del software Gephi: el caso de AIDER en el fortalecimiento del capital social para el Manejo Forestal Comunitario en la cuenca de Aguaytía, Ucayali, Perú
}

\author{
Claudia P. Motta VG. ${ }^{1}$, Marta L. Tostes V. ${ }^{2}$ \\ claudia.motta@pucp.pe, mtostes@pucp.edu.pe \\ ${ }^{1}$ Main Researcher, EXCEDESA S.A.C., Av. Los Ingenieros 570, 12, Lima, Perú \\ ${ }^{2}$ Main Professor at the Academic Department of Management Sciences, Pontificia Universidad Católica del \\ Perú, Av. Universitaria 1801, 32, Lima, Perú
}

DOI: 10.17013/risti.19.131-144

\begin{abstract}
Resumen: La metodología aplicada en la investigación recoge información de tipo cualitativa (entrevistas y observación) y utiliza el software libre Gephi para la construcción y el análisis de la red de organizaciones alrededor del MFC en una localidad de la selva peruana. El caso estudiado fue el proyecto de capital social de la organización no gubernamental AIDER para mejorar el Manejo Forestal Comunitario en la cuenca de Aguaytía. Este análisis propone la medición del capital social y obtiene como resultado la cuantificación del rol intermediario que desempeña la ONG, que permite proponer una línea base de capital social para el proyecto.
\end{abstract}

Palabras-clave: Enfoque de redes; Gephi; capital social; manejo forestal comunitario; ONG

Methodology based on a network approach and the use of the Gephi software: The case of AIDER in strengthening social capital for the Community Forest Management in the basin of Aguaytia, Ucayali, Perú

\begin{abstract}
The research methodology collects qualitative information (interviews and observation) and uses Gephi, a free software, for the construction and analysis of the organizations' network around CFM in a district in the Peruvian jungle. The case of study is the project of social capital from AIDER in order to improve the Community Forest Management in the basin of Aguaytía. This analysis suggests measuring a qualitative concept such as social capital and gives as a result the role played by this NGO and a baseline for the social capital project.
\end{abstract}

Keywords: Network analysis; software Gephi; social capital; community forest management; NGO 


\section{Introducción}

En el Perú, alrededor de 12 millones de hectáreas de bosque se encuentran en territorio que es propiedad de comunidades nativas (Nalvarte Armas, 2015).

El presente artículo se basa en una investigación realizada como parte de una tesis de bachillerato, la cual asume como caso de estudio un proyecto de Manejo Forestal Comunitario (MFC) que viene implementando la Asociación para la Investigación y el Desarrollo (AIDER) en la región de Ucayali, Perú. Esta investigación y la metodología aplicada fueron presentadas durante el $5^{\circ}$ Congreso Ibero-Americano en Investigación Cualitativa y el 1st International Symposium on Qualitative Research que se realizó en la ciudad de Oporto en el mes de Julio del presente año (Motta \& Tostes, 2016). Se agradece las sugerencias realizadas en el marco del Grupo de investigación en gestión de la innovación del Departamento Académico de Ciencias de la Gestión de la Pontificia Universidad Católica del Perú.

Hoy en día se han dado grandes avances en el ámbito de la investigación cualitativa, aunque se enfrenta a nuevos retos frente a la investigación cuantitativa, en especial respecto a su diversidad metodológica y se sugiere la combinación de distintas herramientas de investigación, tanto cualitativas como cuantitativas que han resultado en diseños mixtos (Costa, Reis \& de Souza, 2014).

Para el presente estudio se utilizó una metodología de enfoque de redes que partió del recojo de información cualitativa y, después de su procesamiento en el software libre Gephi, se obtuvo resultados de índole cuantitativo. En este sentido, se centró en la identificación de los vínculos entre los actores y análisis de una red de organizaciones relacionadas al MFC en la provincia de Padre Abad, región Ucayali. Para ello, el primer paso fue la clasificación del mapeo de actores y el levantamiento de información cualitativa por medio de entrevistas tanto a los involucrados en el proyecto como a organizaciones del entorno. Luego, esta información fue ingresada como data al software Gephi, el cual permitió graficar la red y obtener las medidas de densidad, centralidad e intermediación. Como resultado de la investigación, se pudo evaluar la validez de la hipótesis vinculada al relevante rol de intermediación que cumple AIDER dentro de la red de organizaciones del entorno de esta comunidad. La investigación no sólo obtuvo datos concluyentes acerca de los principales puntos vinculados a la hipótesis, sino que también permitió plantear una propuesta de línea de base para el proyecto de capital social.

Esta investigación es un claro ejemplo de la necesidad que existe de identificar y comprender aspectos que no son cuantificables inicialmente, pero se facilita su valoración por medio del software. En este sentido, varios investigadores empezaron a darse cuenta de que el uso de herramientas apropiadas de análisis sobre el contexto físico y social en el que ocurren acciones y comportamientos asociados, permite la estructuración de categorías y su interpretación pertinente a los objetivos planteados para evaluar fenómenos complejos (Costa, Faria \& Reis, 2015).

El esquema del artículo incluye el marco teórico de la investigación, y luego la presentación del caso de estudio y de la metodología que se utilizó. Además, se revisa los resultados obtenidos con el programa Gephi y se propone una línea de base para el proyecto. Por último, se puntualiza las conclusiones finales de la investigación que facilita un entendimiento de la articulación de los actores en el entorno analizado. 


\section{Marco Teórico}

La investigación se basa en tres macro temas que se tratarán a continuación. El primero lo constituye el capital social, concepto que no puede ser entendido desvinculado de la "innovación social". Para la CEPAL, la innovación social se entiende como "nuevas formas de gestión, de administración, de ejecución, nuevos instrumentos o herramientas; o nuevas combinaciones de factores orientadas a mejorar las condiciones sociales y de vida en general de la población de la región" (CEPAL, 2016: 2). Este enfoque toma en cuenta la participación de la comunidad y los beneficiarios, quienes se transforman en protagonistas de su propio desarrollo que debe ser sostenible en el tiempo y replicable en otros contextos.

En este sentido, mientras la innovación social facilita la generación de valor, el capital social pertenece a la dimensión sistémica de su acumulación que analiza los vínculos entre los agentes (Echevarría, 2008). Entre los autores pioneros en el tema, se destaca Bourdieu (1985), sociólogo para quien el capital social se define como el conjunto de recursos reales o en potencia a los que se puede acceder gracias a la posesión de una red de relaciones institucionalizadas en mayor o menor medida y de reconocimiento mutuo. Sin embargo, es con Putnam (1993) que el concepto adquiere relevancia, pues lo define como una serie de aspectos de las organizaciones sociales, que consisten en redes, confianza y normas, las cuales permiten que se implementen acciones en conjunto para obtener beneficios mutuos. Además, este autor menciona que las actividades económicas se optimizan si se cuenta con un gran stock de capital social, lo cual es fundamental para el caso que se analiza a continuación.

De esta manera, el capital social puede ser considerado como la acumulación del valor social, pero no es un concepto fácilmente cuantificable, aunque existen aproximaciones en escala comparativa (Echevarría, 2008). Por ejemplo, bajo el enfoque de redes, se responde a la necesidad de contar con indicadores de articulación de los actores. Otras mediciones pueden hacerse por varias vías:

- Mediante encuestas de percepción, actitudes y valoraciones (positivas, negativas) de determinadas innovaciones propuestas.

- Mediante estudios de apropiación social de dichas innovaciones, que midan su integración efectiva en la vida cotidiana de las personas, lo que puede concretarse en su uso, y todavía más, en el tiempo de utilización de las innovaciones propuestas.

- Mediante métodos cualitativos, por ejemplo cuestionarios y entrevistas a usuarios relevantes, por ejemplo usuarios expertos.

- Mediante estudios comparativos del grado de apropiación de las innovaciones sociales a lo largo del tiempo. (Echevarría, 2008)

El segundo macro tema consiste en el manejo forestal ejercido desde las comunidades (MFC). En un contexto en el que el ambiente se ha vuelto un tema de prioridad y preocupación mundial, el MRC surge como una respuesta ante la necesidad de un uso sostenible de los recursos del bosque. Así, representa una forma de silvicultura que consiste en el aprovechamiento por parte las comunidades de manera sostenible, lo cual implica que decidan gestionarlos para comercializar desde un manejo planificado comunitariamente (Gerez \& Purata, 2008). Por lo tanto, el MFC tiene como actor principal a las comunidades, que impulsa su empoderamiento y la construcción de capital social. 
Un concepto clave al hablar de MFC es el de sostenibilidad. Esta se debe trabajar desde sus tres dimensiones: la económica, la social y la ambiental. De esta manera, el MFC no solo responde a la conservación de los recursos naturales, sino también al uso de estos de tal manera que generen desarrollo para las comunidades que viven en los bosques.

El tercer macro tema es el de las Organizaciones no gubernamentales (ONG) y su protagonismo en distintos campos de acción del desarrollo sostenible. El contar con instituciones estables con capacidad de actuar en zonas donde el Estado no tiene presencia, genera que se construyan alianzas entre distintos grupos de interés que busquen agilizar los procesos para la participación social y la incidencia en políticas desde los ciudadanos (Andrade \& Fontaine, 2011; Sá et al., 2014). En el caso de los temas forestales, se ha podido observar cómo el rol de las ONG se ha vuelto cada vez más indispensable. No solo aportan con conocimientos técnicos y con el fortalecimiento de las capacidades de las comunidades, sino que también facilitan la relación cona otros actores. Asimismo, las ONG funcionan muchas veces como puentes para que las comunidades tengan acceso a los servicios públicos.

\section{Caso de estudio}

El caso sobre el que se aplica la metodología es el proyecto "Fortalecimiento del capital social y su articulación para el manejo forestal sostenible en el proceso de desarrollo alternativo de la cuenca del Aguaytía, región Ucayali”. Este proyecto, que se inició en el año 2014 y concluye en el 2017, es financiado por la Agencia de los Estados Unidos para el Desarrollo Internacional (USAID) e implementado por AIDER.

El proyecto presenta dos objetivos principales: Por un lado, se aspira a lograr el trabajo cooperativo entre los miembros de las comunidades y caseríos en sus propias actividades productivas y de manejo forestal, y promover a la vez la conservación de la biodiversidad. Por otrolado, se busca facilitar el compromiso dentro de las comunidades para alcanzar un desarrollo colectivo y productivo en base al fortalecimiento de las organizaciones ya existentes.

La población con la que se trabaja está compuesta por los grupos que se ubican alrededor de la cuenca de Aguaytía. El proyecto comprende cinco comunidades nativas cacataibo: Puerto Azul, Puerto Nuevo, Yamino, Mariscal Cáceres y Sinchi Roca. Además, incluye seis caseríos: Nuevo Ucayali, Virgen del Carme, Nuevo Satipo, Nolbert del Alto Uruya, San Juan Bautista y La Unión. También involucra dos organizaciones indígenas: la Organización Regional AIDESEP de Ucayali (ORAU) y la Federación Nativa de Comunidades Cacataibo (FENACOCA).

\section{Metodología aplicada}

Costa y otros autores mencionan que la metodología no es algo estático, sino que se construye o modifica en base a la realidad y la complejidad de los problemas. Es por eso que puede basarse, de manera sistemática y coordinada, en metodologías cualitativas con capacidad explicativa. Sin embargo, también se puede buscar articular con otras metodologías y herramientas de investigación que generen una respuesta más efectiva y real al principal problema de investigación (Costa, Reis \& Souza, 2014). 
Para realizar esta investigación se utilizó una metodología de tipo descriptiva y basada en el enfoque de redes. Según Wasserman y Faust (2013), hay cuatro formas para recoger datos de redes sociales: cuestionarios, entrevistas, observaciones y registros de archivos. No se ha considerado el uso de cuestionarios debido a que la mayoría de entrevistados eran líderes indígenas o jefes de caseríos a los que se les dificultaba su llenado. Por el contrario, se encontraban mucho más abiertos a la observación y al intercambio de experiencias, por lo que la entrevista fue la herramienta principal de levantamiento de información. Por otro lado, se recurrió a la observación durante eventos propios del proyecto, lo que permitió ver cómo se daba la interacción entre los actores participantes. Finalmente, se obtuvo información por medio de los registros de archivos en los que se identificaron actividades que se encontraron presentes o interactuaron dos actores o más.

La primera fase consistió en el mapeo de actores, que básicamente trató de identificarlo que forman parte del contexto de las comunidades y caseríos participantes del proyecto. Como un primer acercamiento se realizó la búsqueda en registros de archivos, para lo cual se acudió a dos tipos de fuentes: las documentales, basadas en la información disponible en los sitios web institucionales o noticias periodísticas; y la información proporcionada por un especialista, que en este caso fue la directora del proyecto de capital social de AIDER. Tomando como referencia estos dos tipos de fuentes, se logró construir una lista de actores y se identificó algunas relaciones entre ellos que serían luego validadas durante el trabajo de campo.

El siguiente paso consistió en el análisis de vínculos entre actores, para lo cual fue de gran ayuda la creación de una matriz de vínculos, que permitió realizar el cruce de su interrelación y registrar la evidencia que se iba identificando. Esta matriz contiene a los mismos actores tanto en la primera fila como en la primera columna, de manera que se puedan apreciar las relaciones de un actor con todos los demás en cada casillero. Para completar la matriz fue necesario recopilar evidencia sobre las relaciones y clasificarlas por su intensidad. En este sentido, se realizó una investigación en bases de datos especializadas, buscando registros de archivos y de experiencias de vínculo entre por lo menos dos de los actores identificados. Al culminar, se pudieron encontrar relaciones de diversa naturaleza e intensidad: apoyo financiero, asistencia técnica, audiencia pública, capacitación, congreso, consulta previa, denuncia, feria, mediación, mesa de diálogo, mesa de trabajo, noticia, pedido, programa, proyecto, reunión, sanción y taller. Estos vínculos fueron validados y complementados con el trabajo de campo, y posteriormente se les asignó un peso según criterios que se explicará posteriormente.

La siguiente parte consistió en la elaboración de las entrevistas, para las cuales se establecieron como ejes temáticos el manejo forestal, el capital social, y las ONG. Sobre el primer tema, tomando como referencia las reflexiones de Sabogal (2008), las preguntas se orientaron a pedir que se explique cuál es la actividad de manejo forestal que uno desempeña, qué factor es el más dañino para los bosques y cuáles son los actores más importantes para la actividad. Sobre el capital social, Putnam (1993) facilitó un enfoque que muestra los aspectos más valorados para generar confianza entre actores y para realizar trabajos en conjunto. Siguiendo la línea de la investigación, se profundizó en qué actores son aquellos en los que se confía más y la motivación para hacerlo. Sobre el tema de las ONG, siguiendo la perspectiva de Bridge y Perreault (2009), se preguntó sobre la eficacia de las acciones que realizan las ONG en cuanto al cuidado de los bosques, cuál es su tipo de 
aporte y cómo se procesa. Al final, se pidió evaluar el desempeño de AIDER en cuanto a la contribución que se percibe recibir de esta organización y la confianza que tienen en ella.

El trabajo de campo se realizó en la región de Ucayali a fines de noviembre del 2015 y tuvo una duración de 13 días. Se visitaron dos comunidades nativas (Yamino y Mariscal Cáceres) y seis caseríos (Nuevo Satipo, Virgen del Carmen, Nolbert del Alto Uruya, San Juan Bautista, La Unión y Nuevo Ucayali). El total de entrevistas realizadas fue de veintitrés, en las que se conversó, entre otros, con representantes de comunidades y caseríos, así como con representantes de organizaciones externas. El propósito fue conocer su perspectiva acerca del manejo forestal y el capital social.

Para complementar las entrevistas se realizaron diversos trabajos de observación durante toda la visita, desde el apoyo en las actividades de AIDER y las coordinaciones para las entrevistas, hasta las visitas a los caseríos y comunidades. Finalmente, se estuvo presente en tres eventos realizados como parte del proyecto: "I Foro - Seminario distrital de capital social y manejo forestal para superar la pobreza", "Taller de evaluación participativa" y "Campaña de sensibilización: Unidos por el manejo y conservación de los bosques en San Alejandro". En base a la información recogida mediante la experiencia en el campo, se pudo proceder a construir la red de actores alrededor del proyecto de capital social en la cuenca de Aguaytía.

La herramienta informática que se utilizó fue Gephi, un software de libre descarga que permite, a partir de información ingresada por medio de una matriz, construir un gráfico de redes de los actores involucrados en un mismo tema o entorno. Además, facilita realizar el cálculo del grado de densidad, de entrada, de intermediación, de modularidad y de cercanía de cada actor en relación a otro y en relación a la red. Una de las características del software Gephi consiste en que permite asignar un valor a cada una de las relaciones entre nodos. Así, la información cualitativa, con variables nominales, va adquiriendo capacidad de valorarse en términos de su intensidad, con la construcción de variables ordinales, de tal forma que se permite clasificar a los actores.

Al hablar de relaciones valoradas, Wasserman y Faust (2013) hacen referencia a ciertas características que se usan para asignar un valor. Estas pueden ser la fuerza o intensidad, la frecuencia de la interacción e incluso las cantidades monetarias o la valoración de la amistad con el actor. Es por ello que, para asignar un peso a cada una de las relaciones, se utilizó dos de las variables mencionadas por los autores: la frecuencia de la interacción y la fuerza de la relación. La frecuencia de la interacción fue definida por la cantidad de vínculos identificados entre actores. Para ello se tomó la matriz de vínculos como principal fuente. Por su parte, la fuerza de la relación se basó en construir una clasificación básica de acuerdo a la información obtenida en el trabajo de campo. Dado que los entrevistados mostraron dificultades para graduar la fuerza de la relación, se tuvo en consideración un sistema básico de solo tres niveles.

\section{Resultados}

A continuación se aprecia los resultados de la investigación en términos de los seis factores de análisis de la intensidad de la construcción del capital social en la cuenca de Aguaytía: densidad, grado, grado de entrada, grado con pesos, intermediación y modularidad. 
La densidad se refiere a la proporción que hay entre los lazos existentes y los lazos posibles. Esta medida da una idea acerca de qué tan conectada se encuentra una red en su interior. En este caso, Gephi arrojó como resultado de 29,9\%, lo cual quiere decir que en la red están conectados menos de un tercio del total de nodos. Esto muestra que aún existen muchos actores que no se encuentran conectados entre sí y que, probablemente, ni siquiera se conozcan. Desde la perspectiva del MFC, esto representa una oportunidad de mejora en la medida que las nuevas conexiones entre nodos abrirían nuevas puertas para poder compartir información y coordinar actividades entre ellos.

Respecto al grado, este se resume en el número total de actores al que un nodo está unido, sin importar si son de entrada o de salida. Gephi ofrece una opción que facilita la visualización de los nodos en el gráfico, los cuales se han distribuido según Force Atlas. Bajo esta opción de distribución, los nodos que están vinculados entre sí se encuentran más cercanos, mientras que los que no tienen relaciones se ubican más lejos. El gráfico resultante se observa a continuación.

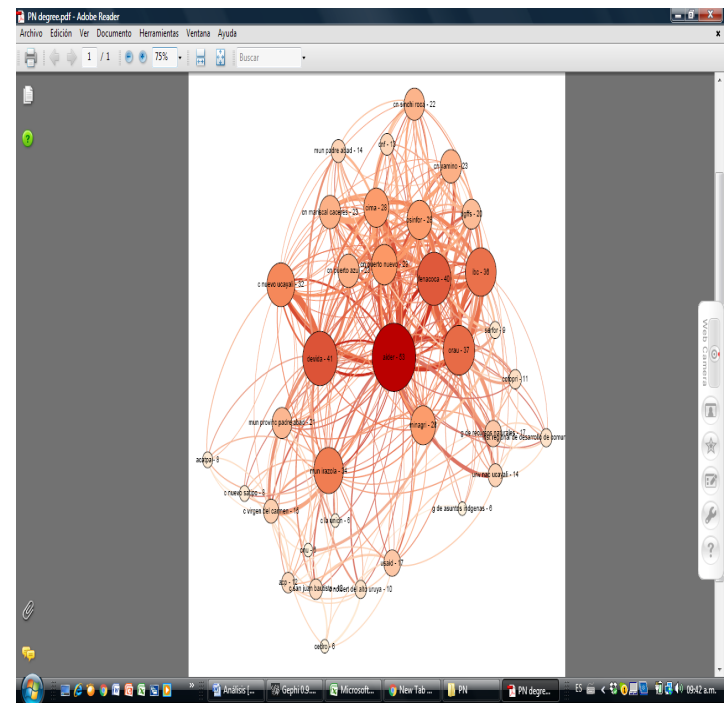

Figura 1 - Gráfico de la red en base al grado. Elaboración propia.

En este gráfico claramente destaca como actor AIDER, con 53 vínculos dentro de la red. Luego se da un salto de más de diez puntos hasta las siguientes mejores organizaciones, que son la Comisión Nacional para el Desarrollo y Vida sin Drogas - DEVIDA (41), FENACOCA (40), ORAU (37), Instituto del Bien Común - IBC (36) y la Municipalidad de Irazola (34). Estos seis actores son los que se encuentran mejor conectados dentro de la red, pues tienen una mayor cantidad de vínculos con otros actores.

Los gráficos anteriores no toman en cuenta el peso de las relaciones, sino solo la cantidad de vínculos que tiene cada uno de los nodos. Gephi permite, para el grado con pesos, asignar un valor a cada uno de los vínculos luego de ingresar la data. En el siguiente gráfico se presenta las relaciones y sus intensidades respectivas reflejadas en los pesos asignados. 


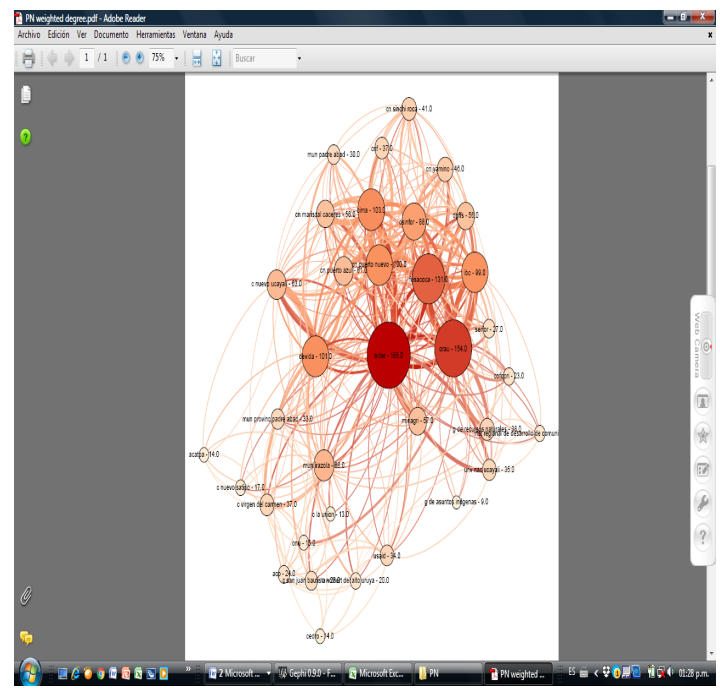

Figura 2 - Gráfico de la red en base al grado con pesos. Elaboración propia.

Para la investigación no fue útil centrarse en el resultado que arroja Gephi, en la medida que este se ve influenciado por la cantidad de relaciones que tiene un actor. Por ello, lo que se hizo fue obtener el promedio de los pesos, lo cual permitió identificar cuáles vínculos tenían mayor intensidad.

Respecto a la intermediación, esta consiste en un factor que se suele asociar directamente con la comunicación entre nodos. El siguiente gráfico muestra el grado de intermediación dentro de la red.

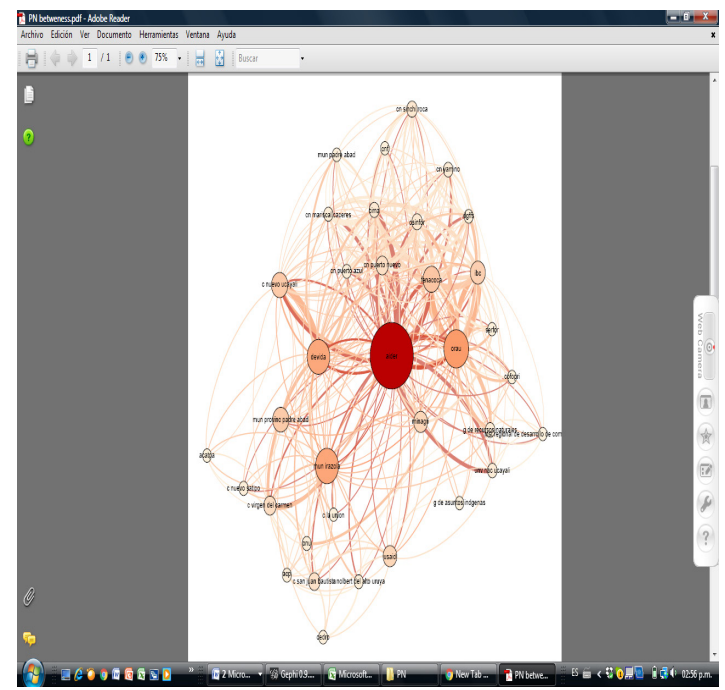

Figura 3 - Gráfico de la red en base al grado de intermediación. Elaboración propia. 
AIDER lidera la lista con 233 puntos. Es el actor que presenta el grado de intermediación más alto, o en otras palabras, muchos nodos se conectan o pasan a través de él para llegar a otro. Esto es lo que se conoce como camino geodésico o la ruta más corta para llegar a otro nodo. Asumir este rol es importante, pues permite la relación de un actor con otro que en circunstancias normales no estaría a su alcance. Los actores que presentan un alto nivel de intermediación facilitan esta dinámica al servir de puente entre dos actores $\mathrm{y}$, a su vez, se adjudican un potencial control de la comunicación entre estos. El alto grado de intermediación de AIDER se debe a que su presencia en la región de Ucayali, que obedece a los varios proyectos que viene implementando, le ha dado la oportunidad de poder contactarse con la mayoría de los actores existentes alrededor del MFC. Esto ha resultado en una oportunidad para AIDER como organización, y una ventaja para las comunidades y caseríos con los que trabaja.

Por su parte, la modularidad es una medida que sirve para identificar comunidades de actores dentro de la red, es decir, grupos de nodos que se encuentren agrupados. El programa detectó cuatro comunidades.

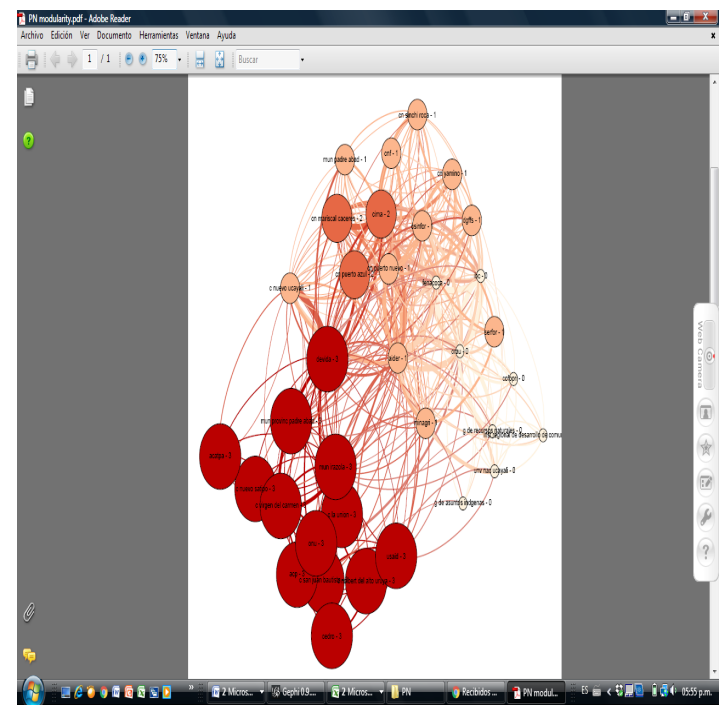

Figura 4 - Gráfico de la red en base a la modularidad. Elaboración propia.

La comunidad con el color más destacado es la que se encuentra alrededor de los caseríos, e incluye a las ONG y asociaciones que están trabajando con ellos, así como algunas instituciones del gobierno. Como se mencionó, este grupo gira alrededor de los caseríos, lo que indica que hay más vínculos y actividad en relación a este grupo. Esto puede vincularse con la importancia que se le está dando últimamente al cacao en la zona y las distintas iniciativas, tanto públicas como privadas, que se están llevando a cabo para poder sacar este sector adelante. 


\section{Propuesta de línea de base}

En base a la investigación, se puede afirmar que el proyecto de capital social implementado por AIDER tiene el potencial para no solo mejorar el manejo forestal de la zona, sino para además proporcionar sostenibilidad a las iniciativas propuestas. Sin embargo, aún existen algunos puntos pendientes a trabajar que pueden ser desarrollados en futuras investigaciones. Teniendo como objetivo el fortalecimiento del capital social, se presenta a continuación una línea de base planteada para el proyecto de AIDER, que fue validada de manera participativa con los involucrados de la organización, tomando en consideración el alcance del proyecto. Los siguientes cuadros hacen énfasis en cada indicador, mencionando a los actores involucrados (organizaciones), las medidas actuales, las medidas esperadas y las acciones sugeridas o a reforzar.

\begin{tabular}{lllll}
\hline Indicador & Organización & $\begin{array}{l}\text { Medida } \\
\text { Actual }\end{array}$ & $\begin{array}{l}\text { Medida } \\
\text { Esperada }\end{array}$ & $\begin{array}{l}\text { Acciones sugeridas / Acciones a } \\
\text { reforzar }\end{array}$ \\
\hline & & & $\begin{array}{l}\text {-Generar más espacios de diálogo entre las } \\
\text { instituciones del gobierno y los caseríos y } \\
\text { comunidades }\end{array}$ \\
Densidad & Toda la red & $29.9 \%$ & $40 \%$ & $\begin{array}{l}\text {-Insertar a comunidades y caseríos en } \\
\text { programas de la municipalidad o del } \\
\text { gobierno regional }\end{array}$ \\
\hline
\end{tabular}

Tabla 1 - Línea de Base - Densidad

Así, se plantea como meta lograr concretar el $40 \%$ del total de conexiones posibles. Para lograr ese 10,1\% restante se sugiere realizar y/o reforzar tres acciones. En primer lugar, se propone fomentar más espacios de diálogo entre instituciones del ámbito público y caseríos, comunidades, asociaciones y federaciones. También se sugiere un trabajo de priorización por actores en el que se identifique cuáles son aquellos que presentan un mayor interés y disposición para un trabajo conjunto con una población determinada. Finalmente, se busca conectar más el trabajo de las municipalidades con los pobladores de sus jurisdicciones, identificando programas en los que las comunidades y caseríos puedan insertarse.

\begin{tabular}{lllll}
\hline Indicador & Organización & $\begin{array}{l}\text { Medida } \\
\text { Actual }\end{array}$ & $\begin{array}{l}\text { Medida } \\
\text { Esperada }\end{array}$ & $\begin{array}{l}\text { Acciones sugeridas / Acciones a } \\
\text { reforzar }\end{array}$ \\
\hline \multirow{5}{*}{ Grado } & $\begin{array}{l}\text { C. Virgen del } \\
\text { Carmen }\end{array}$ & 9 & 20 & \\
\cline { 2 - 4 } & $\begin{array}{l}\text { C. San Juan } \\
\text { Bautista }\end{array}$ & 8 & 20 & $\begin{array}{l}\text {-Identificar actores importantes y encontrar } \\
\text { puntos de trabajo en conjunto dentro del } \\
\text { contexto cacaotero de los caseríos } \\
\text {-Aprovechar la experiencia del Caserío Nuevo }\end{array}$ \\
\cline { 2 - 4 } $\begin{array}{l}\text { C. Nolbert del } \\
\text { Alto Uruya }\end{array}$ & 8 & 20 & Ucayali \\
\cline { 2 - 4 } & C. Nuevo Satipo & 5 & 20 & \\
\cline { 2 - 4 } C. La Unión & 4 & 20 &
\end{tabular}

Tabla 2 - Línea de Base - Grado

Mientras que las comunidades nativas tienen un grado de por encima de 20, los caseríos solo llegan a 9. Esto puede tornarse contraproducente si como consecuencia lleva al 
aislamiento. Es por ello que se ha trazado el grado de 20 como meta a la que se debería llegar, por ser el menor valor obtenido por las comunidades nativas. La acción sugerida conlleva identificar a los actores dentro del contexto cacaotero que estén interesados y dispuestos a realizar un trabajo conjunto. En este punto cabe mencionarse que una parte del trabajo puede ser facilitada gracias a un caserío que no se encuentra en este cuadro. Este caserío, Nuevo Ucayali, posee un alto grado (31) que ha conseguido gracias a la proactividad de uno de sus líderes, quien se desempeña como Secretario de la Asociación Agropecuaria de Productores de Cacao. De esta manera, se cuenta ya con un modelo a seguir no solo en términos de actitud, sino también de la cantidad de contactos con los que cuenta.

\begin{tabular}{|c|c|c|c|c|}
\hline Indicador & Organización & $\begin{array}{l}\text { Medida } \\
\text { Actual }\end{array}$ & $\begin{array}{l}\text { Medida } \\
\text { Esperada }\end{array}$ & $\begin{array}{l}\text { Acciones sugeridas / Acciones a } \\
\text { reforzar }\end{array}$ \\
\hline \multirow{6}{*}{$\begin{array}{l}\text { Grado } \\
\text { promedio con } \\
\text { pesos }\end{array}$} & FENACOCA & $3 \cdot 3$ & 4 & $\begin{array}{l}\text {-Dar seguimiento a los planes de trabajo } \\
\text { - Buscar conexiones con organismos } \\
\text { públicos }\end{array}$ \\
\hline & OSINFOR & 3.1 & 3.5 & \multirow{3}{*}{$\begin{array}{l}\text {-Facilitar el diálogo para hacer entender los } \\
\text { procesos de negociación y la normativa }\end{array}$} \\
\hline & SERFOR & 3.0 & 3.5 & \\
\hline & DGFFS & 2.8 & 3.5 & \\
\hline & Municipalidades & $2.1 / 2.0$ & 2.5 & $\begin{array}{l}\text {-Encontrar puntos de trabajo en conjunto } \\
\text { entre municipalidad, comunidades nativas } \\
\text { y caseríos }\end{array}$ \\
\hline & $\begin{array}{l}\text { Comunidades } \\
\text { Nativas }\end{array}$ & $<2.5$ & 2.5 & $\begin{array}{l}\text {-Potenciar capacidades de negociación y } \\
\text { capacitar en procesos de negociación }\end{array}$ \\
\hline
\end{tabular}

Tabla 3 - Línea de Base - Grado promedio con pesos

La primera organización que se menciona es la FENACOCA. Se reconoce el potencial que tienen dentro de la red por su grado de intermediación, y sin embargo, actualmente no poseen una buena reputación. Por ello, se propone hacer un seguimiento de los planes de trabajo de la organización para garantizar que se cumplan sus objetivos de acuerdo a los plazos establecidos.

Entre las organizaciones públicas se identificaron las siguientes como las más importantes: la Dirección General Forestal y de Fauna Silvestre (DGFFS), el Servicio Nacional Forestal y de Fauna Silvestre (SERFOR) y el Organismo de Supervisión de los Recursos Forestales y de Fauna Silvestre (OSINFOR). Estos son los entes reguladores de toda la actividad forestal en la región Ucayali. Si bien el proyecto no pretende tener algún tipo de injerencia sobre estos actores, se debe mencionar que el diálogo entre estos y las comunidades y caseríos puede facilitarse gracias a AIDER. Tanto para la Municipalidad de Padre Abad como para la de Irazola se plantea una meta parecida: se sugiere buscar la concertación de reuniones en las que participen ambos lados (población y Estado), con la finalidad de identificar objetivos comunes y trazar planes de trabajo en conjunto.

Finalmente, se plantea una acción para las comunidades nativas y caseríos: se busca centrar la atención en los procesos de negociación, de manera que se puedan realizar coordinaciones de manera asertiva, no solo con actores externos, sino también con los grupos de interés internos. 


\begin{tabular}{|c|c|c|c|c|}
\hline Indicador & Organización & $\begin{array}{l}\text { Medida } \\
\text { Actual }\end{array}$ & $\begin{array}{l}\text { Medida } \\
\text { Esperada }\end{array}$ & $\begin{array}{l}\text { Acciones sugeridas / Acciones a } \\
\text { reforzar }\end{array}$ \\
\hline \multirow{6}{*}{ Intermediación } & DEVIDA & 94.1 & 100 & \multirow{3}{*}{$\begin{array}{l}\text {-Mostrar la importancia de ser actores } \\
\text { puentes } \\
\text {-Buscar puntos de coincidencia para } \\
\text { trabajo en conjunto con AIDER }\end{array}$} \\
\hline & CIMA & 8.3 & 50 & \\
\hline & IBC & 42.7 & 50 & \\
\hline & FENACOCA & 55.8 & 100 & -Conectar con el sector público \\
\hline & ACATPA & 0.6 & 50 & \multirow{2}{*}{$\begin{array}{l}\text {-Conectar con actores públicos y con } \\
\text { ONG que ayuden en el proceso de } \\
\text { comercialización }\end{array}$} \\
\hline & $\mathrm{ACP}$ & 0.6 & 2.8 & \\
\hline
\end{tabular}

Tabla 4 - Línea de Base - Intermediación

La FENACOCA se incluye en esta lista porque se espera que este tipo de organización logre un grado de intermediación cercano al de AIDER, de modo que pueda desempeñarse como actor puente. Es por ello que se sugiere reforzar el trabajo que actualmente viene realizando FENACOCA y fomentar el diálogo de esta organización con los organismos públicos.

Se ha planteado una sugerencia común para DEVIDA, Centro de Conservación de Investigación y Manejo de Áreas Naturales (CIMA) e IBC, en la medida que ejercen su rol de manera similar. En primer lugar, se propone dar a conocer los resultados de la presente investigación, donde se muestra que son actores intermediarios importantes, con el fin de lograr que se comprometan con este rol. En segundo lugar, se aspira a buscar puntos de coincidencia en sus proyectos para que se pueda llegar a un trabajo en conjunto y coordinado con AIDER. Estos actores resultan claves para el mejoramiento del manejo forestal y la búsqueda del desarrollo de los pobladores, por lo que es importante que los esfuerzos no se hagan por separado, sino de una manera unificada.

\section{Conclusiones}

Según los resultados de la investigación, se pudo concluir que la ONG AIDER cumple el rol de actor puente entre las poblaciones con las que trabaja y los servicios públicos. De esta forma, se han llegado a crear vínculos importantes para lograr el empoderamiento de las comunidades y caseríos, lo cual permite disminuir su dependencia de actores externos y lograr la sostenibilidad de las acciones llevadas a cabo.

Durante todo el desarrollo de la investigación se buscó generar operatividad en un sentido teórico y en vinculación a la conformación de redes, desde la perspectiva del capital social. Para esto, el uso del software Gephi como principal herramienta de análisis fue vital, pues brindó los insumos para construir una línea de base para el proyecto denominado "Fortalecimiento del capital social y su articulación para el manejo forestal sostenible en el proceso de desarrollo alternativo de la cuenca del Aguaytía, Región Ucayali”.

Si bien los resultados se limitan al campo de acción del caso de estudio, es la metodología la que se rescata para ser replicada en otros contextos que lo requieran. La metodología aplicada responde a una necesidad de análisis cuantitativo en base a información obtenida de manera cualitativa. De este modo, las entrevistas llevadas a cabo con los distintos actores dentro de la red pudieron ser sistematizadas y reflejadas en esquemas 
que facilitaron la cuantificación de los grados de intermediación, centralidad, densidad y modularidad. Por tanto, el uso del software Gephi resultó ser muy enriquecedor para la obtención de las conclusiones de la investigación sobre las hipótesis planteadas inicialmente en relación al fortalecimiento del capital social.

Finalmente, se puede concluir que bajo el enfoque de redes que utiliza el proyecto, y partiendo del recojo de información cualitativa, se han logrado brindar resultados cuantitativos y con una presentación visual amigable, lo cual aumenta las posibilidades de comunicación con los involucrados con vistas a la generación de una perspectiva de desarrollo sostenible para la región.

\section{Referencias}

Andrade, K., \& G. Fontaine (2011). Gobernanza en tres dimensiones: de los recursos naturales, la conservación en áreas protegidas y los pueblos indígenas. Gobernanza ambiental en Bolivia y Perú. Quito: FLACSO, Sede Ecuador, UICN.

Costa, A., B. Faria \& L. Reis (2015). Investigacao através do Desenvolvimiento: Quando as Palavras "Contam". RISTI - Revista Ibérica de Sistemas de Tecnologias de Informação, (E4), vii-X.

Costa, A., L. Reis \& F. Souza (2014). Investigacao Qualitativa para Sistemas e Tecnologías de Informacao. Revista Ibérica de Sistemas de Tecnologias de Informacao, (E2), ix-xii.

Cepal. Org (2016). Acerca de innovación social. Comisión Económica para América Latina y el Caribe. [online] Recuperado el 13 de octubre $2016<$ http://www.cepal. org/es/acerca-de-innovacion-social>

Bourdieu, P. (1985). The forms of Capital. En J. Richardson (ed.) Handbook of Theory of Research for the Sociology of Education, 241-258. New York: Greenwood.

Bridge, G., \& T. Perreault. (2009). Environmental Governance. En N. Castree, \& D. Demeritt, A Companion to Environmental Geography, 475-497. Oxford: Black Well Publishing.

Durston, J. (2000). ¿Qué es capital social comunitario? Santiago de Chile: CEPAL.

Echevarría J. (2008). El Manual de Oslo y la Innovación Social. En J. Sebastián (coord.) ARBOR Ciencia, Pensamiento y Cultura, 732, 609-618. Madrid: CSIC.

Gerez, P., \& S. Purata. (2008). Guía Práctica Forestal de Silvicultura Comunitaria. México: SEMARNAT, CONAFOR, CCMSS.

Motta, C., \& M. Tostes (2016). El rol de AIDER en el fortalecimiento del capital social para el Manejo Forestal Comunitario en la cuenca de Aguaytía: metodología basada en el enfoque de redes y en el uso del software Gephi. En A. Costa (ed.) Atas do $5^{\circ}$ Congresso Ibero-Americano em Investigacao Qualitativa, 3, 720-729. Porto: Ludomedia

Nalvarte Armas, J. (2015). Manejo Forestal Comunitario para mitigar cambio climático: La experiencia de la Comunidad Nativa Callería, Ucayali - Perú. Lima, Perú: Asociación para la Investigación y Desarrollo Integral - AIDER. 
Putnam, R. (1993). Making Democracy Work: Civic Traditions in Modern Italy. Princeton: Princeton University Press

Sá, F., Rocha, Á., \& Cota, M. P. (2014). Quality models for online e-Government services. In 2014 9th Iberian Conference on Information Systems and Technologies (CISTI) (pp. 1-5). IEEE.

Sabogal, C., W. Jong, B. Pokorny, \& B. Louman. (2008). Manejo Forestal Comunitario en América Latina: Experiencias, lecciones aprendidas y retos para el futuro. Belém, Brasil: Una publicación conjunta de CIFOR - CATIE.

Wasserman, S., \& Faust, K. (2013). Análisis de redes sociales: métodos y aplicaciones. Madrid: CIS- Centro de Investigaciones Sociológicas. 